(do) https://doi.org/10.37843/rted.v9i2.136

\title{
Método Socrático en el Aprendizaje Activo de Filosofía de las Estudiantes de Educación- UNSCH 2019
}

\author{
Socratic Method in the Active Learning of Philosophy of Education Students- UNSCH \\ 2019
}

Adolfo Quispe-Arroyo ${ }^{1}$

\section{ఠ}

EDICIÓN: 曲E-CIVTAC

Recibido: 12/mayo/2020

Aceptado: 18/junio/2020

Publicado: 25/septiembre/2020

1Perú

\section{IIIIInstitución \\ ${ }^{1}$ Universidad Nacional de San Cristóbal de Perú}

\section{Correo Eletrónico}

'adolfo.quispe@unsch.edu.pe

\section{(D)ORCID}

'https://orcid.org/0000-0001-5814-5593

\section{Citar así: CSAPA / IEEE}

Quispe-Arroyo, A. (2020). Método Socrático en el Aprendizaje Activo de Filosofía de las Estudiantes de EducaciónUNSCH 2019. Revista TecnológicaEducativa Docentes 2.0, 9(2), 16-23. https://doi.org/10.37843/rted.v9i2.136

A. Quispe-Arroyo, "Método Socrático en el Aprendizaje Activo de Filosofía de las Estudiantes de Educación- UNSCH 2019", RTED, vol. 9, n. ${ }^{\circ}$ 2, pp. 16-23, sep. 2020.

\section{Resumen}

El método o diálogo socráticos es un método de dialéctica o parla lógica para la indagación de nuevas ideas o temas filosóficos en general y de sus disciplinas en particular. La presente investigación se concretó con el objetivo de determinar la influencia del método socrático en el aprendizaje activo de filosofía de las estudiantes de Facultad de Ciencias de la Educación-UNSCH 2019. En este sentido, se realizó una investigación explicativa de enfoque cuantitativo en el paradigma positivista con métodos experimental y comparativo para determinar la influencia de una variable independiente sobre otra variable dependiente. El método socrático en el aprendizaje activo de filosofía, cuyo espacio geográfico corresponde a la Facultad de Ciencias de la Educación de la Universidad Nacional de "San Cristóbal de Huamanga", dicha muestra quedó conformada por 48 estudiantes de la Escuela Profesional de Educación Inicial de dicha Facultad de serie 100 o primer año, curiosamente conformada solo por mujeres, formándose dos grupos a criterio del investigador: uno de control y otro experimental. Los resultados refieren luego de aplicación del método socrático al grupo experimental se incrementó significativamente el índice promedio de aprendizaje activo de filosofía a comparación de aquellas que no fueron expuestas a este método. En conclusión, el método socrático influye significativamente en el aprendizaje activo de filosofía en el grupo experimental a comparación del grupo de control quienes no aplican dicho método en la Facultad de Ciencias de la Educación, según el parámetro estadístico universal "T" de Student $(?<0.05)$.

Palabras clave: Método socrático, aprendizaje activo, filosofía.

\section{Abstract}

The Socratic method or dialogue is a method of dialectic or logical parley for the investigation of new ideas or philosophical themes in general and of their disciplines. The present research was carried out with the objective of determining the influence of the Socratic method in the active learning of philosophy of the students of the Faculty of Education Sciences-UNSCH 2019. In this sense, an explanatory research with a quantitative approach was carried out in the paradigm positivist with experimental and comparative methods to determine the influence of an independent variable on another dependent variable. The Socratic method in the active learning of philosophy, whose geographical space corresponds to the Faculty of Education Sciences of the National University of "San Cristóbal de Huamanga", said sample was made up of 48 students from the Professional School of Initial Education of said Faculty of series 100 or first year, curiously made up of only women, forming two groups at the discretion of the researcher: one control and the other experimental. The results refer after applying the Socratic method to the experimental group, the average index of active philosophy learning significantly increased compared to those who were not exposed to this method. In conclusion, the Socratic method significantly influences the active learning of philosophy in the experimental group compared to the control group who do not apply this method in the Faculty of Education Sciences, according to the universal statistical parameter "T" of Student $(?<0.05)$.

Keywords: Socratic method, active learning, philosophy. 
El método o diálogo socráticos es un método de dialéctica o parla lógica para la indagación de nuevas ideas o temas filosóficos en general y de sus disciplinas en particular. Este método fue aplicado para el análisis de los conceptos morales claves. Fue detallado por Platón en los diálogos Socráticos. Por esto, Sócrates es habitualmente reconocido como el padre de la filosofía moral en Occidente. Al respecto Dynnik (1962), señala:

\begin{abstract}
El método socrático consiste en una dialéctica de los conceptos, en el arte de descubrir las contradicciones en que se incurren las opiniones del adversario, contraponiendo con ese unas dichas opiniones. Este método consta de dos dimensiones fundamentales: la ironía y la mayéutica (por lo que toca a su forma) y la inducción y la definición (por lo que se refiere al contenido). El método socrático es todo un interrogativo. Tendiente a que el interlocutor se contradiga así mismo a fin de que reconozca su propia ignorancia. (p.96).
\end{abstract}

Así mismo Paúl \& Elder (1997) consideran que "la enseñanza socrática es la estrategia educativa más antigua, y aun la más poderosa para promover el pensamiento crítico. Con ella nos enfocamos en formular preguntas a los estudiantes en vez de darles respuestas..." (p. 1).

Es una forma de búsqueda de verdad filosofal. El método socrático implica a dos participantes en cada turno, con uno liderando la discusión y el otro asintiendo o concordando a ciertas presunciones que se le exponen para su aceptación o rechazo. Este método es propio del maestro Sócrates, quien entabló dichos debates con sus acompañantes atenienses en Grecia antigua.

Barylko (2005), al respecto menciona:

\begin{abstract}
El gran invento de Sócrates fue la libertad íntima. La libertad es el pensamiento del individuo. Pensar en qué consiste vivir y cómo hacer para lograr una existencia feliz. Olvidarse de las verdades aprendidas y buscar la razón de ser dentro de uno mismo. Eso enseñó Sócrates. No enseñó a saber. Enseñó a pensar (p.17).
\end{abstract}

Un diálogo socrático puede presentarse en momentos y circunstancias diferentes entre dos hombres o más con actitudes filosóficas o simples curiosidades de temas diferentes en base a preguntas y respuestas de la consulta requiriendo la participación activa que implica esfuerzo, voluntad, reflexión y razonamiento. Se empieza con todo tipo preguntas hasta que los detalles del ejemplo son evidenciados para ser luego usados como plataforma para alcanzar valoraciones más generales.

La práctica del método socrático implica perpetrar una serie de preguntas de un problema, tema o idea central, para responder las otras interrogaciones o series de preguntas incisivas que van generándose durante el diálogo. Este método se emplea para defender una idea en contra de otras con el fin de generar polémicas o diálogos entre los interlocutores. La mejor forma de evidenciar el acierto de un punto de vista es hacer que el oponente mismo encuentre su contradicción y reconozca tal error.

Así mismo, Laercio (2010) se refiere al método del maestro Sócrates como una forma dinámica divertida y sencilla de sacar a luz el conocimiento que se encuentra en uno mismo, es decir el maestro pregunta y el discípulo responde entrando en contradicciones hasta percatarse de su ignorancia. Pasando de esta forma de oscuridad de ignorancia a la luz del conocimiento; por ello también Sócrates comparaba su método con el arte de su madre quien era una comadrona, cuya labor era ayudar a las gestantes a dar a luz del nuevo ser.

En el método socrático es frecuente recurrir a la ironía y mayéutica, por ello hacemos referencia a estas presentaciones del método socrático. El método socrático también se conoce como "ironía socrática". La ironía es la primera de las fórmulas utilizadas por el filósofo griego Sócrates en su dialéctica o discusiones. El maestro Sócrates promueve sus diálogos filosóficos, pedagógicos y propedéuticos a partir de una posición ficticia o imaginario que enarbola al interlocutor, en este caso el discípulo, como el sabio en la materia a tratar o temas a discutir. Sócrates fue considerado como el sabio de Atenas de aquellos tiempos, sin embargo, el pensador solía repetir "sólo sé que nada sé" es fácil entender el porqué de la ironía. Para muchos otros que no reconocían la ironía socrática, el maestro fue muy incisivo que terminaba avergonzando a sus interlocutores.

Al respecto Barylko (2005) sustenta:

Sócrates, en su conversación, en su delicado decir en sus preguntas, desliza la ironía. Ironizar quiere decir afirmar algo sugiriendo, no obstante, que esa 
afirmación oculta otro significado, y que debe ser revisada. La ironía consiste en hacer comprender al otro, de un modo muy especial, lo contrario de lo que se está afirmando. Si le digo a alguien: “iqué lindo peinado ¿Dónde te lo hiciste? ¿Me recomendarías ese peluquero?", podría tratarse de una afirmación veraz de lo que pienso o siento. Pero dicho con tono irónico, con cierta leve y sutil sonrisa (visible y contenida, mi afirmación seguramente le ayudará a darse cuenta de que su peinado es todo lo contrario de lo que yo digo que es. Así funciona la ironía como método para despertar al prójimo en diversas situaciones vitales. (p.21).

El maestro Sócrates con su ironía se contrapone a la opinión infundada o laxa llena de arrogancia de la persona dogmática que cree poseer la verdad sin sospecha o consciencia de su ignorancia. Hacer preguntas bajo la apariencia de tener en alta estima de aparente admiración del saber exhibido por el interlocutor, mostraban, en realidad, la inconsistencia de este y ponían al interlocutor en la disposición de reconocer su ignorancia y error. Algunas veces, el interlocutor, puede que se haya sentido avergonzado o hasta ridiculizado que pudo haber terminado en animadversión hacia Sócrates. Con ironía, Sócrates intentaba minar incisivamente el obstáculo para llegar a la "verdad" que creía poseer el hombre común apoyándose en las ideas triviales y prejuiciosas. A este ejercicio filosófico se creía con derecho Sócrates.

El maestro partía reconociendo su ignorancia. Es famosa su expresión "Sólo sé que nada sé". Según narra Platón en Apología a Sócrates, cuando preguntado el oráculo sobre quién era el más sabio de los griegos, respondió que Sócrates. El maestro lo interpretó en este sentido: que él no era altanero y era simplemente un hombre con ignorancia y aceptaba tal error.

La ironía es lo contrario de actuaciones sofistas: éstos cobraban un dinero a cambio del saber ofrecido; Sócrates no cobraba nada y empezaba por quitarle el saber que creía tener uno. Sócrates comparaba la sofística o labor de los sofistas con el arte culinario, que busca satisfacer

La ironía es lo contrario de actuaciones sofistas: éstos cobraban un dinero a cambio del saber ofrecido; Sócrates no cobraba nada y empezaba por quitarle el saber que creía tener uno.

Sócrates comparaba la sofística o labor de los sofistas con el arte culinario, que busca satisfacer el paladar, pero no se inquieta de las nutriciones; mientras que su propio método se compara con la medicina, pues no se cuida de si causa dolores al paciente, con tal de restituir su salud. La otra dimensión viene a ser la mayéutica como el siguiente paso del diálogo, esto es ayudar a sacar del interior o de psique aquello que el interlocutor sabe, pero ignora.

Para ello el método socrático sugiere realizar preguntas sencillas sobre el tema donde el estudiante o discípulo ha sido nombrado como sabio. Sin embargo, las respuestas que el interlocutor proporcionaba a Sócrates eran refutadas con más interrogantes incisivas con la finalidad de que el interlocutor o el discípulo descubriera que su "saber" era un agregado de prejuicios y opiniones laxas que requerían ser completados y precisados por sí mismo, luego de tomar consciencia, previo reconocimiento de su error y/o ignorancia.

Dynnik (1962), concluye al respecto:

La ironía se completaba con la mayéutica o arte de dar la luz con ayuda del cual el interlocutor nace de nuevo, o sea conoce lo universal en cuanto al fundamento de la verdadera moral. Al decir de Sócrates la duda filosófica "sólo sé que nada sé" debe conducir al autoconocimiento al conócete a ti mismo. Pero lo cierto es que esa duda quebrantada la confianza en la cognociblidad del mundo y servía de instrumento a Sócrates y a sus discípulos para luchar contra el materialismo...con la fundamentación de su método contribuyó en cierto grado a la elaboración de la dialéctica del concepto... (p.96).

La mayéutica es el arte de ayudar a dar a luz la verdad. Consiste en conducir la conversación o la discusión de modo que pueda aflorar la verdad del interior de cada uno. Por otro lado, consideramos el aprendizaje activo en sí uno de los objetivos fundamentales en filosofía, puesto que aprender a filosofar a partir de las diferentes interrogantes sobre la naturaleza, sociedad y el pensamiento para tratar de responder o esclarecer las dudas que todo ser racional conlleva por su existencia temporal como trágica en el sentido filosófico.

El diálogo filosófico, constituye una de las formas del aprendizaje activo del mundo, indubitablemente con ello se está filosofando. La finalidad del diálogo es tocar al interlocutor de las interrogantes propias de todo ser humano de manera abierta y dinámica para poder extraer en el ser activo conciencia de su ignorancia mediante el diálogo con 
el maestro.

El aprendizaje es elemental en una actividad académica, más aún cuando se trata en un nivel superior como es la educación universitaria; este aprendizaje puede manifestarse activa o pasivamente en el aprendizaje de diferentes áreas del conocimiento. En esta oportunidad, para el trabajo hemos optado por el método socrático en el aprendizaje activo de filosofía.

El aprendizaje activo es un aprendizaje basado en motivación, atención y trabajo constante del estudiante, es decir, el estudiante no es un pasivo limitado a escuchar al educador y llenarse de apuntes solamente, sino es considerado como un sujeto central activo y responsable directo de su aprendizaje.

El aprendizaje activo se basa en el estudiante, no en el maestro, no basta escuchar teorías o enseñanzas para aprender, además debemos hacerlo involucrándonos y comprometiéndonos con nuestro aprendizaje de manera activa y responsable, puesto que es nuestra la actividad dinámica. Existe un conjunto de tratados sobre aprendizaje activo, principalmente de enfoque psicológico como pedagógico, sin embargo, no es finalidad de investigación tratar la teoría a profundidad sobre el aprendizaje activo.

No obstante, es necesario sintetizar, para tal fin tomamos referencia los estudios de Sierra (2013) asumiendo como aprendizaje activo a las estrategias que propician una actitud activa del estudiante en clase, en oposición con lo que ocurre en método clásico o tradicional, donde el estudiante se limita a tomar apuntes solamente de lo que observa en la pizarra o de algunas proyecciones. Es el proceso que compenetra a los estudiantes a realizar actividades, así como a pensar en esas cosas ejecutadas.

Para el aprendizaje activo, los estudiantes deben hacer mucho más que oír; deben: leer, cuestionar, escribir, discutir, aplicar conceptos, utilizar reglas y principios, además de resolver problemas.

El aprendizaje activo implica al estudiante estar expuesto continuamente, bien sea por voluntad propia o porque la estrategia utilizada por el educador así lo exige, a situaciones que le demanden operaciones intelectuales de orden superior: análisis, síntesis, interpretación, inferencia y evaluación.

El estudiante es activo y responsable del proceso de aprendizaje. Es consciente de lo que aprende, lo que debe aprender y de lo que aún no ha aprendido. Los estudiantes ponen más énfasis en el desarrollo de habilidades, aprenden a aprender para aprender a hacer $\mathrm{y}$ a ser. Tienen motivación, desarrollan habilidades de orden superior $y$ se sienten competentes para transferir lo que ha aprendido a problemas y escenarios nuevos.

El aprendizaje activo se sustenta en la teoría constructivista del aprendizaje, para el constructivismo los estudiantes son el eje como protagonistas del proceso mismo. Son ellos quienes deciden cuándo, cómo, incluso dónde quieren aprender, mientras el docente hace el papel de un guía: orienta, motiva y retroalimenta a los estudiantes.

Para los ejercicios socráticos en el aprendizaje activo de filosofía referimos a Brenifier (2007), cuyo subtítulo: preguntar, argumentar, profundizar. Facilitan algunas pautas de los ejercicios a tomar en cuenta, ya que si hay un principio fundamental que en nuestro caso queremos inculcar es el reflejo del cuestionamiento, cuestionar al otro, cuestionarse a uno mismo, cuestionar todo lo que es enunciado. Y hay un acceso privilegiado al cuestionamiento: el ¿por qué?, elemento dinámico y detonante, fundador del pensamiento y el discurso, que proporcionará al pensamiento y al discurso su sustancia, pidiéndoles un fundamento y una profundidad.

Los estudiantes captan bien el alcance del ¿por qué?, vemos que una vez iniciados a su uso, cuando tienen que plantear una pregunta, se apresuran a utilizarlo sin parar, a diestro y siniestro, como una solución fácil: ¿por qué dices eso? Porque si los ¿Cuánto?, ¿Cuándo?, ¿Cómo?, ¿Dónde?, ¿A quién?, ¿Cuál?, ¿Qué? o ¿Es esto es x?, entre otras interrogantes que giran al torno de la filosofía. Platón llama a ese proceso en el cual se compromete cada participante a través del cuestionamiento, el principio anagógico. Platón llama a ese proceso en el cual se compromete cada participante a través del cuestionamiento, el principio anagógico.

Por lo referido se planteó la siguiente interrogante de investigación ¿El método socrático influye en el aprendizaje activo de filosofía de las estudiantes de Facultad de Ciencias de la EducaciónUNSCH 2019, cuyo objetivo fue determinar la influencia del método socrático en el aprendizaje activo de filosofía de las estudiantes? En atención a la hipótesis de investigación: el método socrático influye significativamente en el aprendizaje activo de filosofía de las estudiantes de Facultad de Ciencias de la Educación-UNSCH 2019. 
El presente estudio tuvo una relevancia teórica y práctica, metodológica y didáctica como propuesta para la aplicación del método socrático en el aprendizaje activo de filosofía, una alternativa y revaloración del método de un gran maestro de occidente, Sócrates.

La población teórica de esta investigación estuvo conformada por 480 estudiantes, es decir todos los estudiantes de la Facultad de Ciencias de la Educación de la Universidad Nacional de "San Cristóbal de Huamanga" (UNSCH), población muestreada unos 180 estudiantes de toda la Escuela de Formación Profesional de Educación Inicial, la muestra estuvo conformada por 48 estudiantes de Escuela Profesional mencionada, curiosamente integrada de solo mujeres, siendo el tipo de muestreo no probabilístico a criterio del investigador con un diseño experimental en su variante cuasi experimental con pos prueba, formándose dos grupos: uno de control, otro experimental.

El método empleado en el presente estudio fue experimental y comparativo, por presencia de una variable manipulada con la existencia de dos grupos. Los resultados indican influencia significativa del método socrático en el aprendizaje activo de filosofía en el grupo experimental (puntajes mayores) a comparación del grupo control (puntajes menores). En consecuencia, se acepta la hipótesis de investigación, según el parámetro estadístico universal " $T$ " de Student $(\rho<$ 0.05).

\section{Metodología}

El presente estudio se realizó bajo el tipo de estudio: aplicada; nivel de investigación: explicativa; diseño: experimental; nivel social, espacial y temporal: estudiantes de Universidad Nacional de "San Cristóbal de Huamanga" Ayacucho, 2019; población objetiva: estudiantes; muestreo: no probabilística a criterio del investigador (48) se formó dos grupos (experimental y de control); criterio de inclusión: estudiantes de Escuela Profesional de Educación Inicial del primer ciclo o serie 100 de la Facultad de Ciencias de Educación; criterios de exclusión: estudiantes de otras series y otras facultades; Método: experimental y comparativo; técnica e instrumento: evaluación de temas filosóficos con su respectivo instrumento prueba de temas filosóficos; técnicas de procesamiento de datos: paquete estadístico Excel 2014, representado en el cuadro.

\section{Resultados}

\section{Cuadro 1}

Índice de aprendizaje activo de filosofía en el grupo control (sin intervención) y experimental (con aplicación del método socrático) de estudiantes de la Facultad de Ciencias de la Educación- UNSCH, 2019.

\begin{tabular}{|c|c|c|}
\hline $\mathrm{N} .{ }^{\circ}$ & $\begin{array}{r}\text { Grupo } \\
\text { Control }\end{array}$ & $\begin{array}{l}\text { Grupo } \\
\text { Experimental }\end{array}$ \\
\hline 1 & 35,5 & 69,0 \\
\hline 2 & 38,5 & 63,0 \\
\hline 3 & 38,0 & 69,0 \\
\hline 4 & 39,0 & 70,5 \\
\hline 5 & 34,5 & 70,0 \\
\hline 6 & 33,0 & 71,0 \\
\hline 7 & 37,0 & 72,0 \\
\hline 8 & 33,5 & 70,5 \\
\hline 9 & 37,0 & 71,5 \\
\hline 10 & 37,5 & 73,0 \\
\hline 11 & 35,5 & 72,5 \\
\hline 12 & 34,0 & 74,5 \\
\hline 13 & 32,0 & 69,5 \\
\hline 14 & 30,5 & 65,5 \\
\hline 15 & 32,5 & 68,5 \\
\hline 16 & 33,5 & 67,5 \\
\hline 17 & 36,0 & 64,5 \\
\hline 18 & 33,0 & 65,0 \\
\hline 19 & 34,0 & 68,0 \\
\hline 20 & 32,5 & 69,0 \\
\hline 21 & 38,5 & 70,5 \\
\hline 22 & 37,5 & 70,5 \\
\hline 23 & 35,5 & 73,5 \\
\hline 24 & 37,5 & 72,5 \\
\hline $\bar{x}$ & 35,2 & 69,6 \\
\hline Mo & 35,5 & 70,5 \\
\hline $\mathrm{S}$ & 2,4 & 2,9 \\
\hline $\mathrm{V}_{\mathrm{m}}$ & 30,5 & 74,5 \\
\hline $\mathrm{V}_{\mathrm{M}}$ & 39,0 & 74,5 \\
\hline
\end{tabular}

Nota. Procesamiento de datos, elaboración propia.

En el cuadro 1 se muestra los puntajes obtenidos por las estudiantes en el aprendizaje activo de filosofía, cuyas valoraciones oscilan entre: 30 puntos como mínimo y como máximo76. Se evidencia que en el grupo control los puntajes son mucho menos a comparación del grupo 
experimental. A partir de estos datos se opera la inferencia estadística para la prueba de hipótesis.

Al comparar las medias de ambos grupos, se halló una significación menor al 5\%. Por lo tanto, se establece diferencia significativa en el aprendizaje activo de filosofía, entre el grupo experimental de estudiantes con el método socrático y el grupo control que no aplica dicho método en la Facultad de Educación. Es decir, hay una influencia significativa del método socrático en el grupo experimental a diferencia del grupo control.

Se concluye que existe diferencia significativa en el aprendizaje activo de filosofía entre el grupo control de estudiantes que no aplican el método socrático $(\overline{\boldsymbol{X}}=35,2)$ y el grupo experimental con aplicación de dicho método $(\bar{x}=69,6)$ de las estudiantes de Educación en la Universidad Nacional de "San Cristóbal de Huamanga" $(\rho<0,05)$, es decir hay una influencia significativa del método socrático en el grupo experimental a comparación del grupo control.

El método o debate socráticos es una vía para la indagación o búsqueda de nuevas ideas, conceptos, inclusive respuestas profundas en la información. Este método fue aplicado ampliamente para el examen de los conceptos morales claves. Fue descrito por Platón en los diálogos socráticos. Por ello, Sócrates es reconocido como el padre de la filosofía moral en el mundo occidental.

El aprendizaje en los estudiantes se presenta de diferentes ritmos y dinámicas, por ello se ubicaron algunos resultados de investigación del método socrático. Así tenemos a Najarro, quien concluye que "el método socrático, como recurso metodológico, influye significativamente en el desarrollo de la criticidad en los estudiantes de economía de UNSCH, 2010"; (2011, p. 79). Aunque su muestra es de otra Escuela Profesional no desdice el carácter influyente de este método en criticidad de los estudiantes. Por otro lado, tenemos a De La Torre (2003) quien describe al método socrático:

Como camino hacia el esclarecimiento de los conceptos, tal como se perfila en los Diálogos de Platón y, particularmente, en el Menón. Se sitúa el constructivismo del aprendizaje en relación con el empirismo y racionalismo. Se analiza cierta pertinencia contemporánea del socratismo y la forma como ha sido uno de los modelos educativos matemáticos del siglo XX (p.1).
Aunque no es tácito en su descripción concluyente, sí enfoca como la base del aprendizaje, así como pertinente su aplicación. Al respecto Dynnik finiquita: "El Método Socrático es todo un interrogativo. Tendiente a que el interlocutor se contradiga así mismo a fin de que reconozca su propia ignorancia..." (1962, p.96).

Así mismo Paúl y Elder señalan: "la enseñanza socrática es la estrategia educativa más antigua, y aun la más poderosa para promover el pensamiento crítico. Con ella nos enfocamos en formular preguntas a los estudiantes en vez de darles respuestas..." (1997, p. 1).

Es una forma de búsqueda de verdad filosofal. Concierne a dos interlocutores según el turno, con uno liderando la discusión y el otro confirmando o concordando a ciertas presunciones que se le muestran para su aceptación o rechazo. Este método se le atribuye a Sócrates, quien empezó a enzarzarse en dichos debates con sus compañeros atenienses después de una visita al oráculo de Delfos. Aunque en términos comunes actuales sería un tipo acucioso, incisivo e irreverente.

Barylko menciona: "Sócrates no enseñó a saber. Enseñó a pensar" (2005, p.17). Un diálogo socrático puede pasar en cualquier momento entre dos personas cuando éstas buscan respuestas a una pregunta, si ésta la admite mediante su propio esfuerzo de reflexión y razonamiento. Se empieza preguntando con todo tipo de preguntas hasta lograr los detalles del ejemplo evidenciados, para luego ser usados como plataforma en pos de alcanzar valoraciones más generales.

La práctica implica una serie de interrogaciones de un tema, problema o idea central, y responder las otras preguntas que aparezcan incisivamente. Este método se usa para salvaguardar un punto de vista u opinión laxa en contra de otra perspectiva. La mejor forma de evidenciar el acierto de un punto de vista u opinión laxa es hacer del oponente consciente para encontrar y reconocer el error o su ignorancia por sí mismo.

Así mismo, Laercio (2002) se refiere al método del maestro Sócrates como una forma dinámica divertida y sencilla de sacar a luz el conocimiento que se encuentra en uno mismo, es decir el maestro pregunta y el discípulo responde o trata de hacerlo entrando en contradicciones para percatarse de su ignorancia.

En otro trabajo de investigación corroborado por Atao (2017), titulado: método socrático en la 
comprensión de textos filosóficos de los estudiantes de la facultad de ciencias de la educación - UNSCH, 2017, concluye: el método socrático influye significativamente en la comprensión de textos filosóficos. Si por otro lado existen críticos del método socrático, porque no cumpliría un orden lógico, no desmerecen su gran influencia en el pensamiento occidental. Se reconoce como uno de los métodos educativos más antiguos del mundo occidental.

Por lo descrito y experimentado, se puede afirmar respecto al método socrático de las bondades siguientes:

1. Permite hacer una lectura reflexiva $y$ crítica de un tema tratado.

2. Plantea temas filosóficos desafiantes comprometiendo a los estudiantes en discusiones profundas con necesidad de solucionar problemas para poder clarificar las ideas.

3. Contribuye a fomentar una cultura de trabajo en equipo a través de su estructura de participantes y líder.

4. Amplía la base de conocimientos teóricos, reflexivos y críticos del estudiante.

5. Enseña a esgrimir temas filosóficos, ayudando a mejorar la comprensión de los temas tratados, así como de temas afines.

6. Enseña a respetar cierta diversidad de ideas.

7. Crea un vehículo de instrucción para implementar e integrar las capacidades de leer, escribir, escuchar y hablar.

8. El maestro interroga y el estudiante responde, se promueve la participación activa del interlocutor.

El método socrático como arte de discusión o debate es utilizado para ayudar a dar a luz el conocimiento del interlocutor quien reconoce su error o ignorancia a medida que va enfrascándose en contradicciones por las interrogantes incisivas del maestro. Esta actividad, desde luego es activa, requiere voluntad, consciencia, reflexión $\mathrm{y}$ razonamiento de los interlocutores.

En la presente investigación se consideró el

método socrático en el aprendizaje activo de filosofía de las estudiantes universitarias formadas en dos grupos, cuyo resultado establece diferencias significativas en el aprendizaje activo de filosofía, entre el grupo experimental de estudiantes con el método socrático y el grupo control que no aplica dicho método.

En conclusión, existen diferencias significativas en el aprendizaje activo de filosofía entre el grupo experimental de estudiantes con el método socrático y el grupo de control que no aplica dicho método en la Facultad de Ciencias de Educación de la Universidad Nacional de "San Cristóbal de Huamanga", es decir hay una influencia significativa del método socrático en el aprendizaje activo de filosofía en el grupo experimental (puntajes mayores) a comparación del grupo control (puntajes menores). En consecuencia, se acepta la hipótesis de investigación, según el parámetro estadístico universal "T" de Student $(\rho<0.05)$.

A partir de los resultados se invoca a la comunidad científica a continuar con las investigaciones de los beneficios de la aplicación del método socrático en el aprendizaje de diferentes áreas del conocimiento. Así como sus estudios desde diferentes enfoques y paradigmas para validar y revalidar la conclusión a la que arribamos. Por los resultados obtenidos se demuestra la influencia del método socrático en el aprendizaje activo de filosofía para ser generalizados en condiciones parecidas y considerar dentro de los métodos a utilizar en la educación universitaria como una revaloración y contextualización del método utilizado por el gran maestro Sócrates.

\section{Referencias}

Abbagnano, N. et al. (1971). La Evolución de la dialéctica. Ediciones Martínez Roca.

Atao Rivas, David (2017). Método socrático en la comprensión de textos filosóficos de los estudiantes universitarios UNSCH, Ayacucho [tesis de maestría no publicada, Universidad de Huamanga]. Repositorio institucional UNSCH. http://biblioteca.unsch.edu.pe/cgibin/koha/opac-MARCdetail.pl?biblionumber $=4264$

Barylko, J. (2001). La filosofía: Una Invitación a Pensar. Editorial Planeta.

Brenifier O. (2007). La naturaleza del filosofar. Consultado el 27 de abril de 2019. http://www.pratiquesphilosophiques.fr/es/blog-es/

De La Torre, A. (2003). Método Socrático y el método Van Hiele. Colombia: Universidad de Antioquía Medellín. 
Dynnik. M. T. (1962). Historia de la filosofía. Editorial Grijalbo.

Laercio, D. (2002). Vidas de filósofos ilustres. Editorial Omega.

Najarro-Rúa, A. (2011). Método socrático en la criticidad de los estudiantes UNSCH, Ayacucho [tesis de maestría no publicada, Universidad de Huamanga] repositorio institucional

UNSCH.

http://repositorio.unsch.edu.pe/handle/UNSCH/1347

Paúl, R. \& Elder, L. (1997). Enseñanza Socrática. Ediciones fundación para el pensamiento crítico.

Platón (1971). Apología a Sócrates. Editorial Eudeba.

Sierra-Gómez, H. (2013). El aprendizaje activo como mejora de las actitudes de los estudiantes hacia el aprendizaje. Ediciones UPNA.

Rosental, M. (2008). Diccionario filosófico. Ediciones Pueblos Unidos. 